\title{
Physical Optics versus Gaussian Beam Shooting for Shadow Field Analysis in High Frequency Regime
}

\author{
Christine Letrou*, Igor Gershenzon ${ }^{\dagger}$, Yaniv Brick ${ }^{\ddagger}$, and Amir Boag ${ }^{\dagger}$ \\ ${ }^{*}$ Lab. SAMOVAR (UMR CNRS 5157), TELECOM SudParis, Evry, France \\ e-mail: christine.letrou@telecom-sudparis.eu \\ ${ }^{\dagger}$ School of Electrical Engineering, Tel Aviv University, Tel Aviv, Israel \\ e-mails: igor1999@gmail.com,boag@eng.tau.ac.il \\ †Institute for Computational Engineering and Sciences, The University of Texas at Austin, Austin, Texas, USA \\ e-mail: yaniv.brick@fulbrightmail.org
}

\begin{abstract}
Simulation of high frequency fields using Iterative Physical Optics and Gaussian Beam Shooting algorithms is studied. Particular attention is payed to the field evaluation accuracy in shadow regions. The accuracy is evaluated by comparing the results to those of numerically rigorous numerical methods.
\end{abstract}

\section{INTRODUCTION}

Scattering from bodies composed of smooth surfaces, in the high frequency regime, can be analyzed by using asymptotic approaches, such as Physical Optics (PO) [1] and Gaussian Beam Shooting (GBS) [2]. In this paper, the ability of these methods to simulate accurately enough the fields in the shadow region of impenetrable obstacles is tested against Method of Moment (MoM) results. The problem of interest is chosen to resemble scattering by buildings, a situation encountered in urban or suburban environments for ground radar or mobile communication applications. At the first stage, the implementation and testing are performed for scalar (acoustic) fields.

The paper is organized as follows. The iterative PO formulation including shadow radiation is reviewed in Section II. In Section III, we review the GBS formulation, again focusing on the shadow radiation. Finally, a comparative study of the PO versus GBS is outlined in Section IV.

\section{ITERATIVE PHYSICAL OPTICS ALGORITHM}

The physical optics (PO) approach provides a favorable trade-off between efficiency and robustness, as compared to numerically exact integral equation methods and ray-based methods, for convex scatterers, as well as for some particular non-convex cases. For non-convex geometries, the incorporation of multiple reflection effects can be achieved by the Iterative Physical Optics (IPO) approach [3-6]. At each iteration of the IPO scheme, a field reflection (often termed "bounce") is evaluated by a radiation integral operating on the previous iteration's induced sources. Each "bounce" field is in turn used to produce a "correction" source distribution. The solution is obtained by the summation of all such induced source distributions. The application of an IPO scheme to realistic geometries at high frequencies involves two major challenges: (i) The high computational complexity involved in the evaluation of radiation integrals from a surface to surface (each IPO iteration requires evaluation of a field integral at an $O\left(N^{2}\right)$ cost, where $N$ denotes the number of quadrature points. (ii) Accounting for non-trivial mutual visibility conditions between points on the scatterer surface.

In this work, the fast iterative PO (FIPO) algorithm in [7] is employed, in order to address both computational challenges. The treatment of the non-trivial mutual visibility is done by using a shadow-radiation mechanism [5], which nullifies the sources induced on non-illuminated regions $[3,5,6]$. That is in contrast to specifically introducing a mutual visibility function (a function determining which surface mesh elements are geometrically visible to each other). The shadow-radiation mechanism also enables the application of fast field integral computation algorithms for the acceleration of various steps of the algorithm as follows: the shadowing is obtained by a nested set of PO iterations; for each bounce, the resulting surface distribution is computed via multiple shadow radiation iterations. Both the shadow iterations and "bounce" iterations take the form of an accelerable field integral. In the proposed formulation, the acceleration is performed by using the multilevel non-uniform grid (MLNG) algorithm-a divideand-conquer strategy that reduces the field integrals' cost and, consequently, the algorithm's computational complexity to $O(N \log N)[4,8,9]$.

As a test case, the IPO algorithm is used to analyze scattering by a target comprising two objects with mutual shadowing depicted in Fig. 1. The scattering cross section of this configuration computed by PO with geometric and integral shadowing, as well as MoM is presented in Fig. 2. One can observe that PO with integral shadowing produces results that are quite close to those of MoM at a substantially lower computational cost.

\section{GAUSSIAN BEAM SHOOTING ALGORITHM}

In GBS algorithms, propagated fields are represented as a superposition of Gaussian beams (GB), which are launched 
from the emitting antenna and transformed through successive interactions with obstacles. The GBS algorithm used in this work is based on the frame decomposition of source fields into a set of spatially and spectrally translated windows constituting a frame [2].

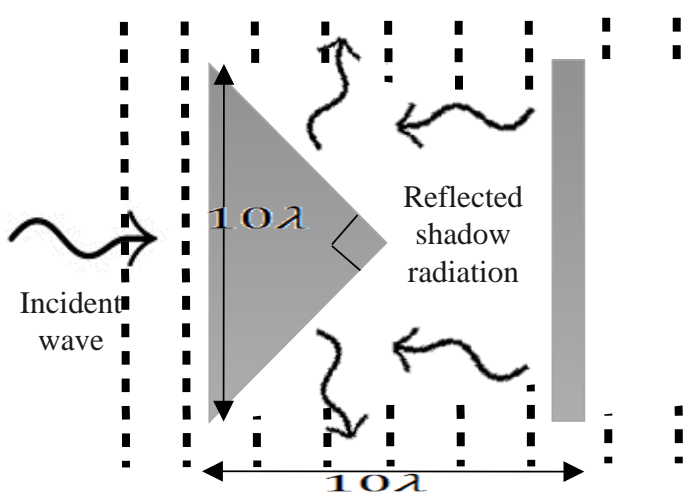

Fig. 1. Test case geometry of a plate hidden by a triangular prism plane section schematic representation. The geometry is uniform and finite in the transverse direction.

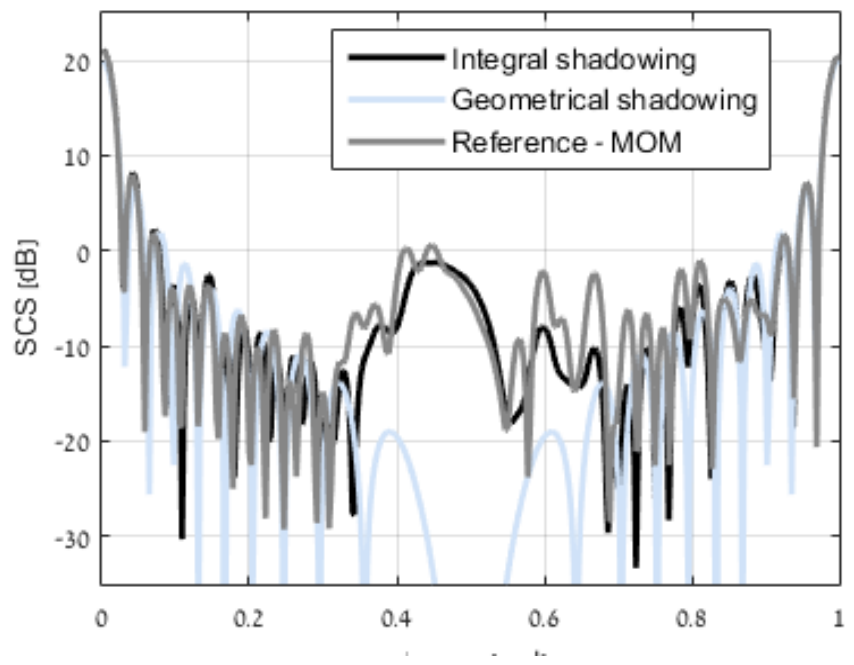

Fig. 2. Scattering cross section computed using PO with two types of shadow field evaluation techniques and MoM for the test geometry depicted in Fig. 1.

Spectrally narrow frame windows radiate in the form of paraxial GBs. GBS algorithms exploit two interesting features of paraxial GBs to accelerate field computations:

- $\quad$ paraxial beam fields can be expressed in the form of complex ray formulas, which allow for fast field computation;

- $\quad$ the transverse localization of paraxial GBs allows to limit the number of beams to shoot through specifically developped selection algorithms determining which beams contribute to the field in a given zone or on a given surface.

In this work, involving non penetrable obstacles, the shadowing approach used by the GBS algorithm is based on two different beam processing methods:

- for beams which are completely "blocked" by an obstacle, only reflected fields are computed, and summed with the fields of the incident beams, propagated in free space ahead of their reflexion ;

- for beams which are only partially blocked by an obstacle, an equivalence principle is applied : the total fields are obtained by superposing fields propagated in free space (without obstacle) and fields radiated by additional source distributions on the surface of the obstacle, designed to satisfy the correct boundary conditions (reflected fields on lit surfaces, nullifying fields on other surfaces). The superposition of all these distributions is considered as a new source distribution on the surface of the obstacle and decomposed on frames defined on this surface.

Any frame-decomposed field distribution being considered as a new source, the same beam processing methods are applied to beams launched from this source. A selection is performed among the beams, either launched from the initial source into free space, or from the new sources on obstacle surfaces, to keep only those reaching the final observation region. The selected beam fields are finally accumulated in this region.

The frame based GBS algorithm has been used, for example, to simulate scattering by a cuboid representing a PEC "building" in the presence of a PEC ground. The test frequency is equal to $430 \mathrm{MHz}$, a frequency used in ground-based UHF radars. A building $20 \mathrm{~m}$ high, with a $5 \times 5 \mathrm{~m}^{2}$ basis, is placed at a distance of $200 \mathrm{~m}$ from the source plane $x=0$. The building is illuminated by a Gaussian beam originating at point $(0,0,12.5)$ and propagating in the $x$-direction. Figure 3 presents the total field in a horizontal plane at a height of $12.5 \mathrm{~m}$. The shadowing effect is visible on the right of the figure. Results have been shown to match with physical theory of diffraction (PTD) and parabolic wave equation results [10].

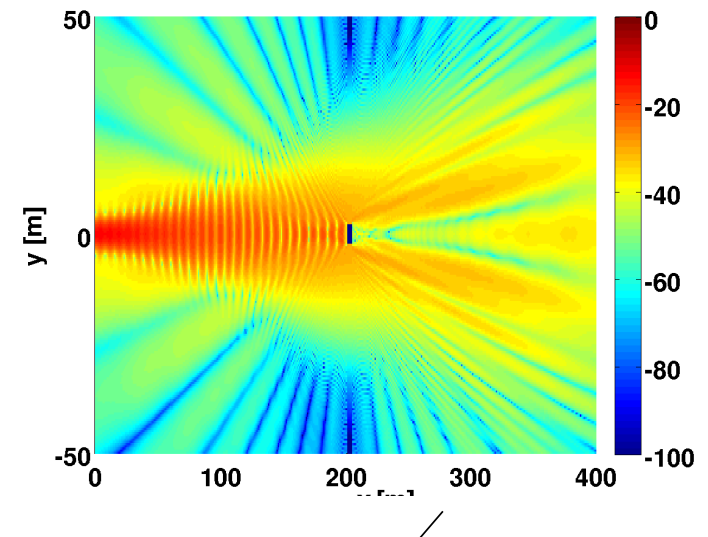

Fig. 3. Field in the horizontal plane computed using GBS for the case of a rectangular building $5 \times 5 \times 20 \mathrm{~m}^{3}$ illuminated by a Gaussian beam at $430 \mathrm{MHz}$.

\section{COMPARATIVE STUDY: PO VS. GBS}

Comparison of the two high frequency methods with MoM serving as a reference will be presented. The comparison will be based on accuracy analysis of both far- and near fields similar to those presented in Figs. 2 and 3, respectively. Of particular interest will be accurate evaluation of weak shadow fields in complex geometries. 


\section{REFERENCES}

[1] P. Y. Ufimtsev, "New insight into the classical Macdonald physical optics approximation,” IEEE Antennas Propag. Mag., vol. 50, no. 3, pp. 11-20, 2008.

[2] A. Shlivinski, E. Heyman, A. Boag, and C. Letrou, “A phase-space beam summation formulation for ultrawide-band radiation," IEEE Trans. Antennas Propagat., vol. 52, no. 8, pp. 2042-2056, 2004.

[3] R. J. Burkholder, C. Tokgöz, C. J. Reddy, and W. O. Coburn, "Iterative physical optics for radar scattering predictions,” Appl. Comput. Electromagn. Soc. J., vol. 24, no. 2, pp. 241-258, 2009.

[4] A. Boag, "A fast iterative physical optics (FIPO) algorithm based on non-uniform polar grid interpolation,” Microw. Opt. Technol. Lett., vol. 35, no. 3, pp. 240-244, 2002.

[5] A. Thomet, G. Kubické, C. Bourlier, and P. Pouliguen, "Improvement of iterative physical optics using the physical optics shadow radiation,” Prog. Electromagn. Research M, vol. 38, pp. 1-13, 2014.

[6] M. F. Cátedra, C. Delgado, and I. G. Diego, "New physical optics approach for an efficient treatment of multiple bounces in curved bodies defined by an impedance boundary condition,” IEEE Trans. Antennas. Propag., vol. 56, no. 3, pp. 728-736, 2008.

[7] I. Gershenzon, Y. Brick, and A. Boag, "Iterative Physical Optics (IPO) with Integral Evaluation of Self-Shadowing," IEEE COMCAS 2015: The International IEEE Conference on Microwaves, Communications, Antennas and Electronic Systems, Tel Aviv, Israel, November 2015.

[8] Y. Brick and A. Boag, "Multilevel non-uniform grid algorithm for acceleration of integral equation based solvers for acoustic scattering," IEEE Trans. Ultrason., Ferroelect., Freq. Control., vol. 57, no. 1, pp. 262-273, 2010.

[9] C. Letrou, V. Khaikin, and A. Boag, "Analysis of the RATAN-600 radiotelescope antenna with a multilevel Physical Optics algorithm," Compte Rendus Physique, vol. 13, pp. 38-45, 2012

[10] E. Fnaiech, C. Letrou, A. Ginestet, and G. Beauquet, "Paraxial Gaussian Beam Shooting algorithm for 3D propagation simulation in built environments," 2015 International Conference on Electromagnetics in Advanced Applications (ICEAA), Turin, Sept 2015, pp. 1155-1158. 